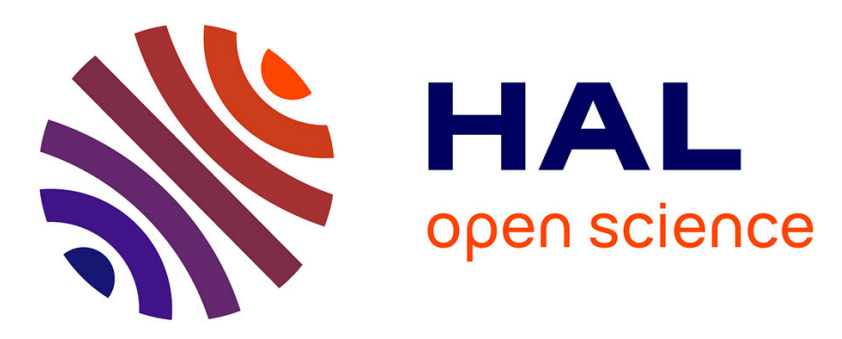

\title{
Knowledge update in adaptive management of forest resources under climate change: a Bayesian simulation approach
}

Rasoul Yousefpour, Jette Bredahl Jacobsen, Henrik Meilby, Bo Jellesmark

Thorsen

\section{To cite this version:}

Rasoul Yousefpour, Jette Bredahl Jacobsen, Henrik Meilby, Bo Jellesmark Thorsen. Knowledge update in adaptive management of forest resources under climate change: a Bayesian simulation approach. Annals of Forest Science, 2014, 71 (2), pp.301-312. 10.1007/s13595-013-0320-x . hal01098415

\section{HAL Id: hal-01098415 https://hal.science/hal-01098415}

Submitted on 24 Dec 2014

HAL is a multi-disciplinary open access archive for the deposit and dissemination of scientific research documents, whether they are published or not. The documents may come from teaching and research institutions in France or abroad, or from public or private research centers.
L'archive ouverte pluridisciplinaire HAL, est destinée au dépôt et à la diffusion de documents scientifiques de niveau recherche, publiés ou non, émanant des établissements d'enseignement et de recherche français ou étrangers, des laboratoires publics ou privés. 


\title{
Knowledge update in adaptive management of forest resources under climate change: a Bayesian simulation approach
}

\author{
Rasoul Yousefpour • Jette Bredahl Jacobsen • \\ Henrik Meilby $•$ Bo Jellesmark Thorsen
}

Received: 6 December 2012 / Accepted: 22 July 2013 /Published online: 6 September 2013

(C) INRA and Springer-Verlag France 2013

\begin{abstract}
- Context We develop a modelling concept that updates knowledge and beliefs about future climate changes, to model a decision-maker's choice of forest management alternatives, the outcomes of which depend on the climate condition.

- Aims Applying Bayes' updating, we show that while the true climate trajectory is initially unknown, it will eventually be revealed as novel information become available. How fast the decision-maker will form firm beliefs about future climate depends on the divergence among climate trajectories, the long-term speed of change, and the short-term climate variability.

- Methods We simplify climate change outcomes to three possible trajectories of low, medium and high changes. We solve a hypothetical decision-making problem of tree species choice aiming at maximising the land expectation value (LEV) and based on the updated beliefs at each time step.

- Results The economic value of an adaptive approach would be positive and higher than a non-adaptive approach if a large
\end{abstract}

\section{Handling Editor: Marc Hanewinkel}

Contribution of the co-authors Rasoul Yousefpour: Corresponding Author, Developing the conceptual model, Analysis of the example, Providing tables and figures, Writing the manuscript, Supervising preparation of the manuscript according to the comments of all co-authors, Setting up the MS according to the format of AFS Jette Bredahl Jacobsen, Henrik Meilby, and Bo Jellesmark Thorsen: Developing the conceptual model and analysis, and writing the manuscript.

R. Yousefpour $(\bowtie) \cdot$ J. B. Jacobsen $\cdot$ H. Meilby $\cdot$ B. J. Thorsen Department of Food and Resource Economics, University of Copenhagen, Rolighedsvej 23, 1958 Frederiksberg C, Copenhagen, Denmark

e-mail: ry@ifro.ku.dk

J. B. Jacobsen • B. J. Thorsen

Centre for Macroecology, Evolution and Climate, University of Copenhagen, Rolighedsvej 23, 1958 Frederiksberg C, Copenhagen, Denmark change in climate state occurs and may influence forest decisions.

- Conclusion Updating knowledge to handle climate change uncertainty is a valuable addition to the study of adaptive forest management in general and the analysis of forest decisionmaking, in particular for irreversible or costly decisions of long-term impact.

Keywords Subjective risk $\cdot$ Belief update $\cdot$ Adaptive forest management $\cdot$ Monte Carlo simulation $\cdot$ Species selection

\section{Introduction}

Central issues in the design of models and methods for adaptive forest management are the uncertainties characterising future climate developments, their impacts on forests, the way we model and describe these uncertainties, and the way we assume or model how decision-makers take into account past, current, and future information. A wide range of decision-maker categories may be considered, including both non-adaptive and adaptive decision-makers, the latter taking into account that more information about climate and forest development is forthcoming and may change the optimal choice, within a continuous decision process (Bolte et al. 2009; Prato 2000; Yousefpour et al. 2012; Schou 2013). Adaptive decision-making in forestry is mostly formulated within the framework of stochastic dynamic programming, which explores a well-defined decision space with welldefined state transition probabilities, possibly decisiondependent, and can determine an optimal solution by breaking the problem down into a sequence of decision steps over time or states and integrates explicitly across uncertain outcomes (Armstrong et al. 2007; Jacobsen and Thorsen 2003).

Uncertainty about future climate change is, however, fundamentally different from many other uncertainties, whose impact on optimal management have been explored at length 
in the literature (see reviews like Yousefpour et al. 2012 and Hildebrandt and Knoeke 2011). Because future climate paths depends on yet unknown decisions, global development and still only partially understood dynamics of the global climate and ecosystems, we cannot credibly model the decisionmakers perception of climate uncertainty as a stationary stochastic transition function. Whereas we may have a reasonable idea, e.g. of the stochastic processes driving prices, costs, interest rates and other uncertainties, this is not the case for climate change.

Therefore, it is perhaps not surprising that the IPCC predictions have described possible climate development in terms of various scenarios, the likelihood of which is rarely described but in qualitative terms.

In the present paper, we adopt a related approach and assume the decision-maker relate to a limited set of possible future climate developments, from the current climate to a new climate regime. The decision-making challenge associated with the development of the changing climate is that while climate may change in the future and be in a different regime than the current, the path followed towards such a possible new climate regime is unknown to us. The new climate regime will perhaps only stabilise slowly — if at all — and the development towards it is characterised by natural variation, which itself may or may not change in scale (Scherrer et al. 2005). This variation will reduce our ability to discern on which trajectory the climate development is and towards which future climate regime. Furthermore, there may be uncertainty about the underlying speed of climate change and the possible characteristics of future climate regimes (Allen et al. 2000; Iverson and Perrings 2012). Thus, any series of future stochastic realisations of climate states must be interpreted by the decision-maker in the light of the best available and updated knowledge and expectations - i.e. beliefs - about the likely new long-run climate regime, the path towards it, and the variation around it.

There are many forestry decisions where adaptive management may be optimally implemented in a fairly reactive manner. If a climate development affects forest productivity, thinning and harvest schedules can be continuously adjusted as the forest state changes. Precautionary management of forest ecosystems is a good example of such a reactive decision for adaptation to the risk of climate change. For instance, Yousefpour and Hanewinkel (2009) examined adaptation to climate change in the Black Forest area of south-western Germany by the conversion of Norway spruce monocultures towards mixed spruce beech forests asking for diversified silvicultural interventions. From a decision-making point of view, this type of decisions are not critical, as long as the future developments are known steady states and the optimal decision can be made now and do not change over time. Therefore, the emphasis of this paper is on forest management decisions where the long-term effects of climate change on forest ecosystems and associated economic outcomes are unknown and likely to change what would be then - in the future-considered optimal decisions now. For example, when choosing tree species in the regeneration phase, attention to the future and long-term climate development is crucial. Due to the nature of climate change uncertainty and the value of knowledge and future information, we propose the Bayesian updating approach (Bayes and Price 1763) as a sound way of improving decisions over time by evaluating new information, analysing alternative decisions and the impacts of current expectations with respect to possible future climate change developments. Bayesian updating is a process where the perceived probability, here called beliefs, of different outcomes is updated in a forward moving process based on the data and observations at hand and prior information (Crome et al. 1996; Kangas et al. 2000; Probert et al. 2010).

We develop a model framework that applies Bayesian updating of beliefs about future climate change in the decision-making process, and otherwise builds on a set of hypothetical forest management outputs under a limited set of possible and different climate change scenarios. Each combination of management alternative and climate scenario may represent a forest management regime with several possible management actions for each climate scenario. Management actions may be optimised contingent on the climate scenario, or may simply be flat simulations. The Bayesian modelling concept enables us to show how a decision-maker may integrate new climate relevant information into his decisionmaking by updating his beliefs about climate change direction (Hauser and Possingham 2008; McDonald-Madden et al. 2010; Prato 2000; Probert et al. 2010). We show that the time needed until the decision-makers beliefs concentrate on the true climate trajectory depends on crucial aspects like the divergence among potential climate trajectories, the speed of change in the long-term regime shift and the short-term climate variability. Finally, we show the potential gain from updating the beliefs by comparing the worst and the best outcomes, respectively.

\subsection{Relation to earlier work}

There is a significant body of research on how various sources and forms of uncertainty and risks can be handled in forest management (for recent reviews of this, see Eriksson 2006; Hanewinkel et al. 2011; Hildebrandt and Knoeke 2011; Yousefpour et al. 2012). As described by Yousefpour et al. (2012), only a limited number of studies address adaptive forest management with climate uncertainty. Many of these rely on simulation analyses to evaluate decision alternatives, and they take little account of the facts that (1) much more will be learned about actual climate change as time passes, and (2) this will allow forest managers to re-evaluate decision alternatives at later stages, which will (3) change their possibilities 
of making optimal decisions to adapt to actual climate development.

We remain inspired by this literature in its approach to deliberately integrate a model of the decision-makers' processing of forthcoming information into the analysis of decision alternatives (McDonald-Madden et al. 2010; Probert et al., 2010; Iverson and Perrings 2012). However, we stress that this literature relies on the ability to define for a given period the stochastic process of relevant variables and their development in the state space. For example, estimates of empirical distributions and data generating processes for prices that can be assumed valid for future prices developments are used to construct transition probability matrixes in closed form, which can be applied validly to assess the expected value of different decision alternatives. Acknowledging that future climate change is inherently uncertain, we therefore resort to Bayesian updating, and draw inspiration from recent studies in the field of biological conservation (McDonald-Madden et al. 2010; Probert et al. 2010). Moreover, it is essential to consider a range of possible changes in climate regimes (low change - high change) and the stochastic nature of changes. This modelling framework is presented in the following with an illustrative example of species selection in forestry.

\section{Material and methods}

\subsection{Models of possible climate trajectories}

Climate development and future climate trajectories are of course complex and multidimensional by nature. For the purpose here, we allow ourselves to represent possible climate changes in a very simplified way. We model climate development using a single-state variable. It could be interpreted as an indicator of climate rather than a single variable like temperature or precipitation. We assume the deterministic part of a given possible climate trajectory from current to future climate regimes can be described with a simple Gompertz timedependent deterministic growth functions (Tomar and Ranade 2002). Furthermore, the annual variation around any trajectory is assumed to be i.i.d (independent and identically distributed) stochastic shocks according to a Wiener noise process with constant variance, $\sigma^{2}$, across state, time, and climate models. The deterministic part of the absolute climate state $x$ for climate trajectory $i$ at time $t$ is given by the model $i$ :

$x_{i t}=x_{i \max } \exp \left(\ln \left(x_{i 0} / x_{i \max }\right) \times \exp \left(-\alpha_{i} t\right)\right)$

$x_{i \max }$ is the maximum possible level of $x$ in climate trajectory $i$, towards which the climate converge and is assumed to stabilise. The initial climate state at $t=0$ is $x_{i 0}$, which is constant across trajectories. The deterministic drift is $\alpha_{i}$, a scaling parameter for regime $i$. We selected a set of values for these constants and parameters, to illustrate a variety of simplified alternative climate trajectories a decision-maker may face, $\mathrm{cf}$. Table 1.

We consider $I$ models of how the climate may develop such that the observed state of the climate, $x_{t}^{o}$ at time $t$ (given the unobserved climate trajectory model $i$ ) is given by:

$x_{t}^{o}=x_{i t}+\varepsilon_{i t} \approx N\left(x_{i t}, \sigma_{i}^{2}\right)$

Where, $t=1, . ., T, i=1, \ldots, I, x_{i t}$ the mean trajectory by model $i$ at time $t$ as given by Eq. (1), and $\varepsilon_{i t}$ is a stochastic variation in climate with normal distribution around the mean 0 and scenario specific variance, $\sigma_{i}{ }^{2}$. As a result, the observed climate state at time $t$ belongs to a noncommittal probability distribution on climate change, i.e., $N\left(x_{i t}, \sigma_{i}^{2}\right)$.

\subsection{The decision-maker's beliefs and processing of information}

We have defined a set of potential climate development scenarios as predicted by the various models, of which only one can be the true in any specific case (if-then analysis). We now set up a decision framework where the decision-maker holds a set of beliefs regarding the probability of each possible climate model $i=\{1, \ldots, I\}$ being the true one. We also show how the decision-maker may change his beliefs using Bayesian updating given and depending on any new observations.

Let $w_{i t}$ be the belief in a particular climate change trajectory, such that beliefs are complete:

$\sum_{i=1}^{m} w_{i t}=1, w_{i, t} \geq 0$ for all $i, t$

Thus $w_{i t}$ is the decisions maker's perceived probability at time $t$ that a climate change trajectory $i$ is the true representative of the climate development $\left\{w_{i t}=\operatorname{Pr}\left(\operatorname{model}_{i}, t\right)\right\}$ given all the information available). As time goes and new knowledge about the climate is obtained through monitoring, as given by $x_{t}^{o}$, the plausibility of each climate trajectory model is

Table 1 Parameters used to simulate slow, medium or fast changes towards low, medium or high future stabilised climate states (Model I-III)

Future climate state

\begin{tabular}{llll}
\cline { 2 - 4 } & High & Medium & Low \\
\hline$x_{\max }$ & 5.00 & 4.00 & 2.00 \\
$\alpha$ & 0.15 & 0.10 & 0.05 \\
$x_{i 0}$ & 0.05 & 0.05 & 0.05 \\
\hline
\end{tabular}


reassessed and the weights, $w_{i t}$, are updated. Here, a complete faith in a model is indicated by $w_{i t}=1$, and $w_{i t}=0$ stands for a null faith. We make use of this information to update our beliefs in each of the alternative models, using Bayes' theorem:

$$
\begin{aligned}
w_{i, t+1}\left(x_{t}^{o}\right) & =\operatorname{Pr}\left(\operatorname{model}_{i} \mid x_{t}^{o}\right) \\
& =\frac{\operatorname{Pr}\left(x_{t}^{o} \mid \operatorname{model}_{i}\right) \operatorname{Pr}\left(\operatorname{model}_{i}, t\right)}{\sum_{i=1}^{I} \operatorname{Pr}\left(x_{t}^{o} \mid \operatorname{model}_{i}\right) \operatorname{Pr}\left(\operatorname{model}_{i}, t\right)}
\end{aligned}
$$

where $\operatorname{Pr}\left(x_{t}^{o} \mid \operatorname{model}_{i}\right)$ is normally distributed with the probability density function:

$$
\phi\left(\frac{x_{t}^{o}-x_{i t}}{\sigma_{i}}\right)=\frac{1}{\sigma_{i} \sqrt{2 \pi}} e^{-\frac{1}{2}\left(\frac{\left(x_{t}^{o}-x_{i t}\right)^{2}}{\sigma_{i}{ }^{2}}\right)}
$$

The weights at time $t+1$ depend on the applied climate model, the alternative models contained in $I$, and the observed climate state $x_{t}^{o}$ at time $t$. The values of $x_{t}^{o}$ could be drawn by Monte Carlo techniques from a normal distribution i.e. $N\left(x_{i t}, \sigma_{i}^{2}\right)$ to simulate particular sequences of climate realisations.

\subsection{The decision-maker's objective}

The decision-maker evaluates the decision alternatives to achieve an objective, which could follow any economic, social, ecological interest or a mixture thereof. We consider a decision-maker with economic interest, who aims at maximising the land expectation value (LEV) of a forest by choosing at each time step the decision alternative in the forest management that implies the best expected LEV, with expectations being based on his beliefs about climate change. The LEV measure can reflect a particular management, which is maximised conditional on the different climate change scenarios being true. This method is applicable to forest management scenarios where a set of sequential decisions must be made and the underlying system dynamics are Markovian. We determine the management action, depending on the objective, time, and the current state of the system. In our problem, the state variable is an information state or belief in each model, $w_{i t}$. For each time step, all possible decisions are evaluated for every possible combination of a discretised set of model weights, $W_{t}=\left\{w_{1 t} ; w_{2 t} ; \ldots ; w_{I t}\right\}$ obtained at each time step using Bayes' theorem (see Eq. 4).

We use $E\left(w_{t}, t\right)$ to denote the expected present value of a management decision, $a_{t j}$, so that the optimal action $a_{t j}$ satisfies:

$$
\max _{a_{i j}} E\left(W_{t}, t\right)=\sum_{i=1}^{I} w_{i t} \operatorname{LEV}_{i t}\left(a_{t j}\right)
$$

The value function $E\left(W_{t}, t\right)$ is the weighted sum of the expected LEVs from action $j$ given model $i$, and the reward received at the decision point $t$. Note that the expectation applies the subjective probability or belief weights, $w_{i t}$, that come from Eq. 4, and it is this updating and combination process that ensures the management is adaptive in nature, in the sense that new decisions will reflect the updated beliefs, taking the novel information into account. Note, however, that the distribution of beliefs (representing probabilities) is not stationary over time, and depends on the specific realisation of the true underlying climate trajectory.

In the real option literature, the concept of the "value of waiting" is used to describe the value of adaptive behaviour compared to a non-adaptive approach. For the Bayesian updating approach, a similar simple measure does not exist, as decision-makers do not know the future probability space and more importantly do not share the same belief of it. Unless the distribution of beliefs in the population of decision-makers is known, little can therefore be said on the average value of waiting. Instead, we illustrate very simply, the value in case the beliefs are most wrong compared to the revealed. This gives a measure of how wrong a decision-maker can be assuming the wrong climate trajectory-within the span of analysed climate regimes.

\subsection{The hypothetical decision problem}

We cast our conceptual model and decision problem in the form of a choice of one among several possible species in a reforestation decision. While this decision problem only occurs once in every rotation in a single forest stand, it occurs continuously at a forest level. It is this continuous decision that we look at. We assume that a decision has to be taken at time $t$. In other words we do not optimise the timing of the decision, and hence do not allow a decision of 'No afforestation'. We consider four species to be selected for a forest plantation in the central European temperate zone. Two species are considered to benefit from future climatic changes. These two species are "climate-winners" and could increase the LEV of plantations if climate change in that direction. These tree species will be more or less reactive to the change, and we assume one with a high $\left(S_{\text {winnerl }}\right)$ and another with a low $\left(S_{\text {winner2 }}\right)$ increase in wood production. In addition, we define one tree species to be a "climate-loser" species which would face a decrease in wood production under climate change $\left(S_{\text {loser }}\right)$, but is superior to both the climate winners, under current climate. Finally, a "climate-indifferent" species is considered as a species which does not respond to the expected levels of climate change at all $\left(S_{\text {indifferent }}\right)$. In the conditions of central Europe, these assumptions may correspond to the performance of European beech (Fagus sylvatica) and Norway spruce (Picea abies) with high $\left(S_{\text {winner1 }}\right)$ and low $\left(S_{\text {winner2 }}\right)$ increase in annual productivity, respectively 
Table 2 Coefficients of the models ( $p$ and $r$ for each model $i$ ) used to predict the LEV (e.g. in $100 € /$ ha) of the future production

\begin{tabular}{llrll}
\hline & $S_{\text {winner } 1}$ & $S_{\text {loser }}$ & $S_{\text {winner2 }}$ & $S_{\text {indifferent }}$ \\
\hline$p$ & 2.0 & 4.9 & 3.5 & 4.7 \\
$r$ & 1.5 & -0.2 & 1.0 & 0.0 \\
\hline
\end{tabular}

(Jacobsen and Thorsen 2003; Spiecker 2003; Yousefpour and Hanewinkel 2009). A good example of loser species $\left(S_{\text {loser }}\right)$ could be Scots pine (Pinus sylvestris) reported to lose productivity and vitality under climate change in central European forests (Sykes and Prentice 1996). Another example of a species perceived as indifferent to climate change $\left(S_{\text {indifferent }}\right)$ would be Douglas fir (Pseudotsuga menziesii) although criticised as being exotic to central European ecosystems (Spiecker 2003).

At the forest level, species selection takes place every time new areas are to be regenerated, and we assume this to happen at all-time intervals $t$. Thus, the decision-maker at each decision point $t$ adjust the species choice to current beliefs about future climate, cf. Eq. (6).

An example may illustrate the decision problem: If environmental changes are assumed negligible and annual volume increment is the same as under the current climate, or climate change is slow and effects on the forest are limited, planting $S_{\text {loser }}$ or $S_{\text {indifferent }}$ may be favoured. Otherwise, if a decisionmaker believes changes will be larger, a favoured action may be to select climate-winner species like $S_{\text {winner1 }}$ or $S_{\text {winner2 }}$. As times passes the decision-maker may change his beliefs, and thus change his preferred choice of tree species to adapt management to perceived futures.

The decision-maker in the model is assumed to know that LEV of species $j$ depends on the climate variable $x_{i t}$ (see Eq. 1) according to the linear model $\operatorname{LEV}_{i t}\left(a_{t j}\right)=p_{j}+r_{j} \times x_{i t}$. We choose a hypothetical set of parameters $(p, r)$ for all $J$ species, and deliberately adjust these to ensure that illustrative patterns of interest will emerge, in particular that all tree species may be the preferred species for some expectations of climate development. The coefficients for the four species are fixed as shown in Table 2.

Applying the parameters of Tables 1 and 2 along with the relations between the deterministic component of climate state development, Eq. (1), and the LEV of each species, we show in Table 3 the resulting hypothetical LEV performance measures for the four species across the three climate scenarios and the three decision points in our model: $2010(t=0), 2020$ and 2030.

Applying the coefficients in Table 3, and three alternative climate models, we simulate the decisions of a forest owner maximising the land value and updating his beliefs on climate development as described above. As each set of realisations (Monte Carlo simulations) is unique and will yield a specific series of beliefs, belief updates, and related decisions, we simulate a large number of realisations and evaluate the typical decision across simulations using MATLAB. In the following, we present the results of predictions, belief updates, the decisions taken with and without Bayesian updating, and the economic consequences of applying such an adaptive decision-making. Moreover, the sensitivity of the updating process to the model's specific variance $\sigma_{i}^{2}$ is analysed and the effects are discussed.

\section{Results}

To assess the change in belief in each climate model and the potential trajectory of the plantations' outputs over the planning horizon, we simulated the implementation of optimal strategies as perceived by the decision-maker, given his beliefs. Simulations were run from relevant starting states with initial priors $\left(w_{1}, w_{2}, w_{3}\right)$, being the beliefs in the different climate models. Monte Carlo simulations were repeated for each climate trajectory model $(100,000$ iterations for each period), defining each one in turn to be the true model from which realisations would be drawn and the decision-maker

Table 3 The level of the deterministic component of the climate state variable and the performance of the four species at these climate states, applying the linear relation between LEV, $x_{i t}$ and using the parameters of Tables 1 and 2

\begin{tabular}{|c|c|c|c|c|c|c|c|c|c|}
\hline \multirow{2}{*}{$\begin{array}{l}\text { Climate model, } i \\
\text { Year, } t\end{array}$} & \multicolumn{3}{|l|}{ I } & \multicolumn{3}{|l|}{ II } & \multicolumn{3}{|l|}{ III } \\
\hline & 2010 & 2020 & 2030 & 2010 & 2020 & 2030 & 2010 & 2020 & 2030 \\
\hline$x_{i t}$ & 0.12 & 0.51 & 1.25 & 0.51 & 2.16 & 3.67 & 1.25 & 3.67 & 4.67 \\
\hline LEV, $S_{\text {winner1 }}{ }^{\mathrm{a}}$ & 2.17 & 2.76 & 3.87 & 2.76 & 5.23 & 7.50 & 3.87 & 7.50 & 9.00 \\
\hline LEV, $S_{\text {loser }}^{\text {a }}$ & 4.88 & 4.81 & 4.68 & 4.81 & 4.51 & 4.24 & 4.68 & 4.24 & 4.06 \\
\hline $\mathrm{LEV}, S_{\text {winner2 }}^{\mathrm{a}}$ & 3.62 & 4.01 & 4.75 & 3.62 & 5.66 & 7.17 & 4.75 & 7.17 & 8.17 \\
\hline LEV, $S_{\text {indifferent }}{ }^{\mathrm{a}}$ & 4.70 & 4.70 & 4.70 & 4.70 & 4.70 & 4.70 & 4.70 & 4.70 & 4.70 \\
\hline
\end{tabular}

$x_{i t}$ the climate variable

${ }^{\mathrm{a}}$ Species performance measured in land expectation value (LEV $\left.100 € / \mathrm{ha}\right)$ 

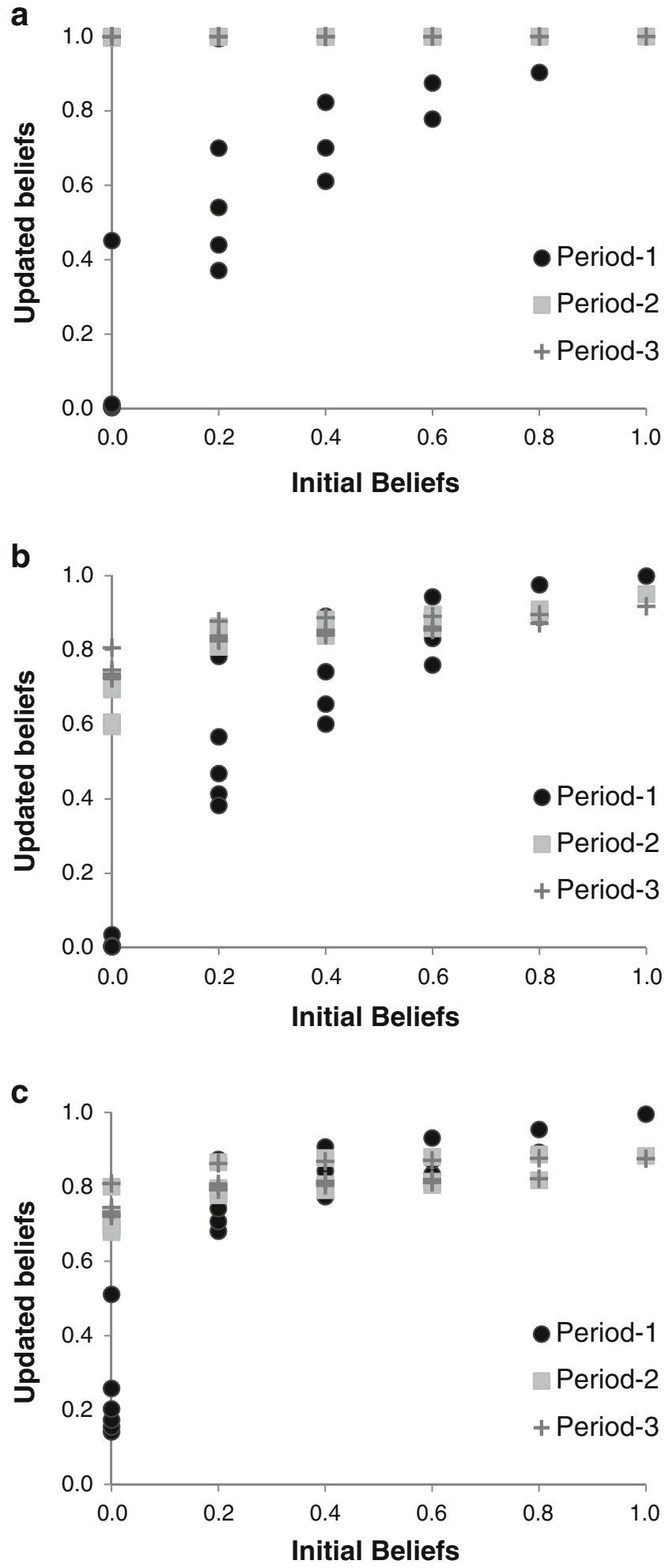

Fig. 1 Update of the belief in the true climate over time, depending on initial beliefs (at 2010=Period-1) and the assumed true climate model I (a), II (b) and III (c)

react to. At each time step, the optimal action, given updated beliefs, was implemented in the plantation area $(s)$ normalised to 1 , given the time steps of current beliefs $\left\{w_{1 ; i} ; w_{2 ; i} ; \ldots\right.$;
oSloser OSwinner2

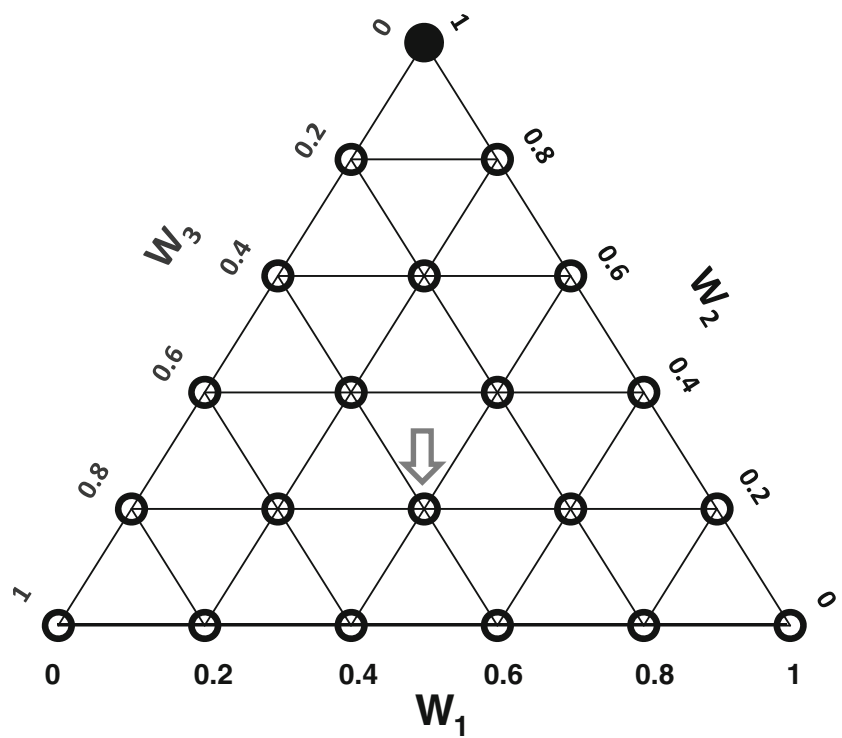

Fig. 2 Species selection depending on initial beliefs $(0-1)$ at the beginning of period 1 (before any updating). Axes of ternary plot illustrate the beliefs in each climate change model ( $W_{1-3}$ for models I-III, respectively). For example, the grey array shows that if the beliefs in climate models IIII are $0.4,0.2$ and 0.4 , respectively, the optimal adaptive decision is $S_{\text {loser }}$ (the hollow black circle)

$\left.w_{3 ; t}\right\}$. Observed climate state $\left(x_{t}^{o}\right)$ were drawn from a normal distribution with the mean given by the underlying true climate model, and a standard deviation of 0.3 (Allen et al. 2000). Based on the observed climate state, the belief in each model was updated (Eq. 4) for the next period, taking annual steps. The process of implementing actions, acquiring climate observations and updating beliefs was calculated in each of the three decision points $(2010,2020$, and 2030).

\subsection{Belief update over time}

In the following, the results of a sensitivity analysis are shown for different sets of initial beliefs, given different assumptions about the true underlying climate model (moderate, medium, and high change in the climate variable, cf. Table 1). Figure 1 shows examples of belief updates and the time needed to recognise the true underlying climate. We have analysed the sensitivity of the procedure to different sets of initial beliefs, with decadal updating and with three decision points distributed over 30 years. Figure 1a shows that for our choice of climate trajectories and variability, just 10 years of observations provide enough information to update our beliefs towards the true climate change scenario, when this is the scenario of moderate change (climate model I). This result shows little sensitivity to the initial beliefs.

Figure 1 shows the corresponding time needed to update our beliefs towards true climate model if medium (Fig. 1b, climate II) or high changes (Fig. 1c, climate III) in climate are expected. Unlike in Fig. 1a, we see that for these trajectories (which are 
a

\section{OSloser OSwinner2 $\square$ Sindifferent}

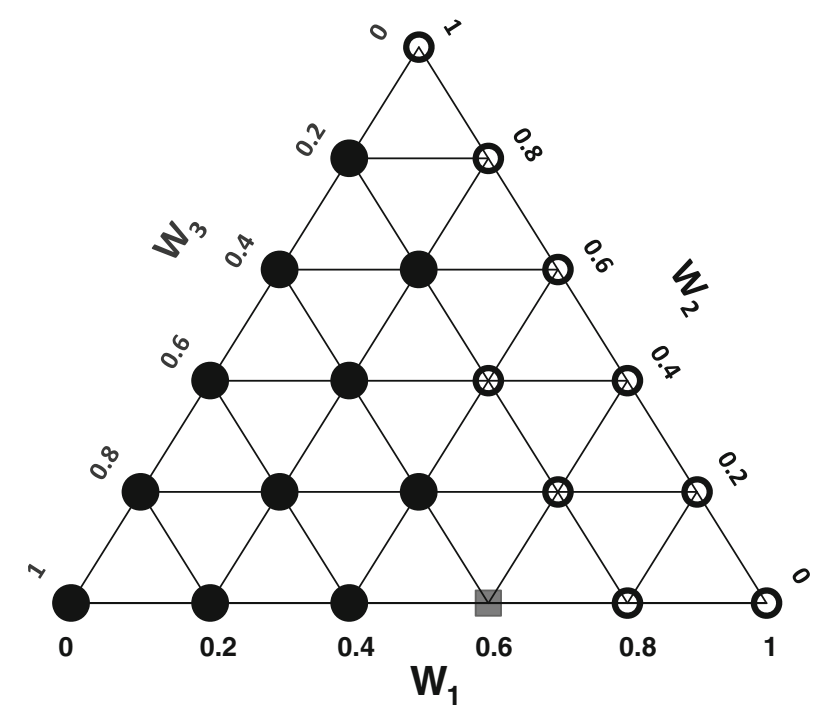

b

Swinner2

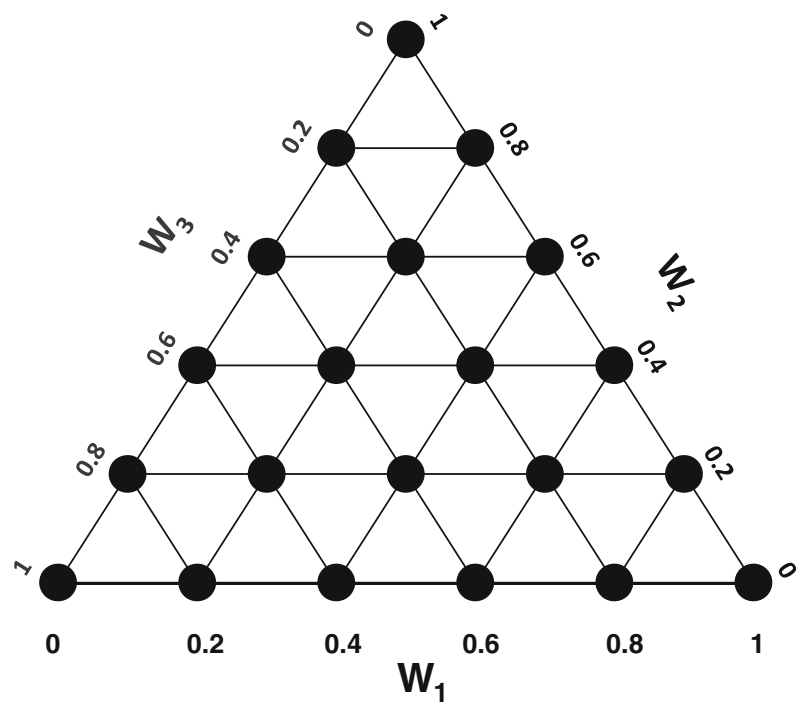

Fig. 3 Species selection depending on initial beliefs $(0-1)$ at $t=0\left(W_{1-3}\right)$ and decision points period 2 (a) and period 3 (b) under low climate change (I)

also designed to be closer to each other, cf. Table 1) there is a need for longer periods of observations for beliefs to converge towards the true underlying climate, and moreover, due to the annual variation, even with 30 years of observations confidence in the true model is not absolute cf. Fig. 1b, c. Initial beliefs play a great role for approaching the recognition of the true climate and the stronger the initial belief in the true model, the faster the belief converges. The process of updating with Bayesian approach is depending on the model's specific variance $\sigma_{i}=0.3$. a
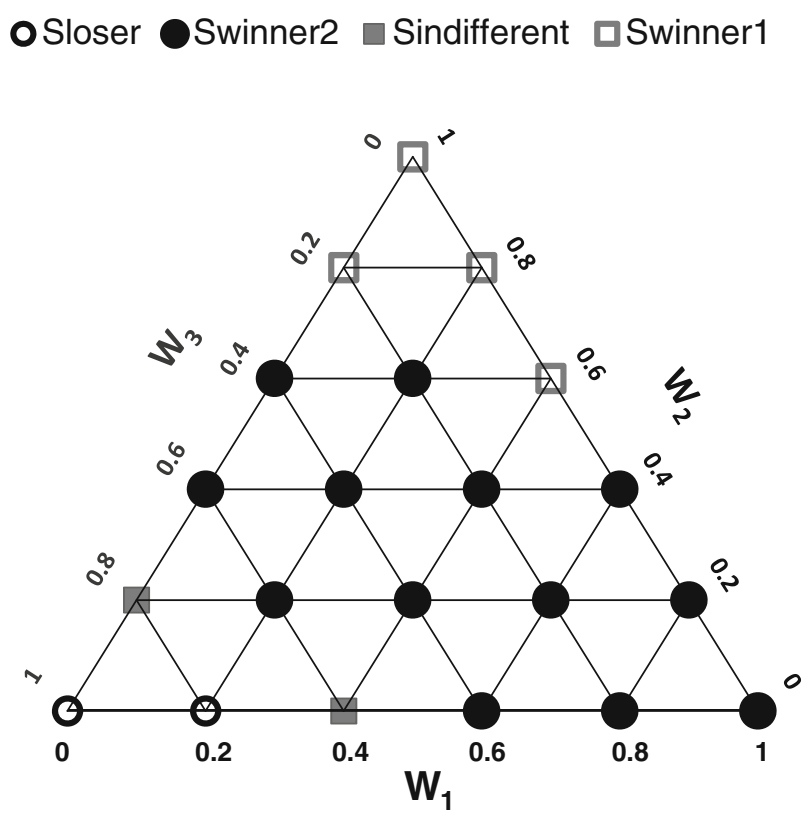

b

\section{口Swinner1}

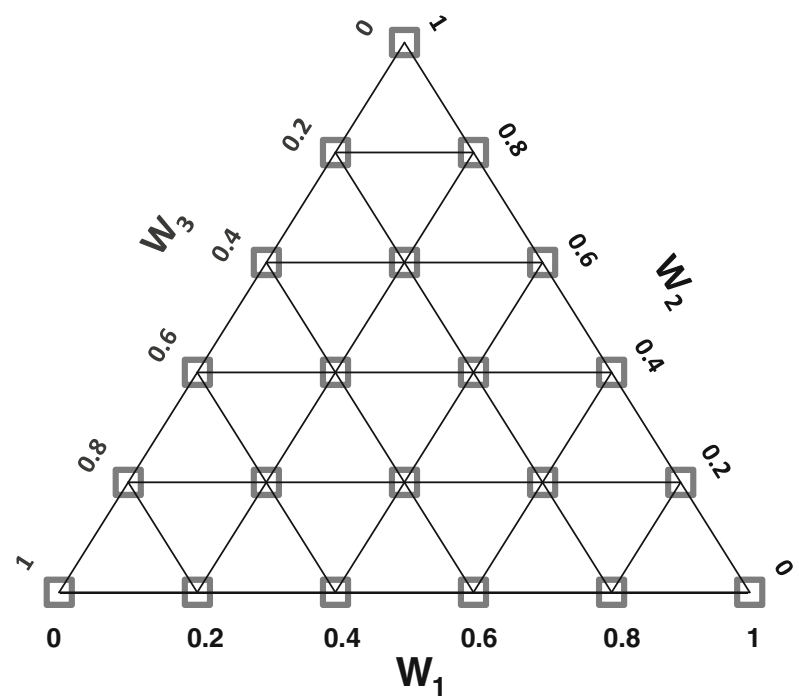

Fig. 4 Species selection depending on beliefs $(0-1)$ at $t=0\left(W_{1-3}\right)$ and decision points; periods 2 (a) and period 3 (b) under medium climate change (II)

Therefore, the sensitivity of this process to a lower $\sigma_{i}=0.1$ or higher $\sigma_{i}=0.5$ variance is analysed and the results are shown in Appendix A. We comment on this point in the discussion.

\subsection{Optimal decision on species selection}

Optimal decision on species selection depends not only on the performance of the species under different climate conditions but also on the decision-maker's beliefs regarding the climate 
a

OSwinner2 $\square$ Swinner1

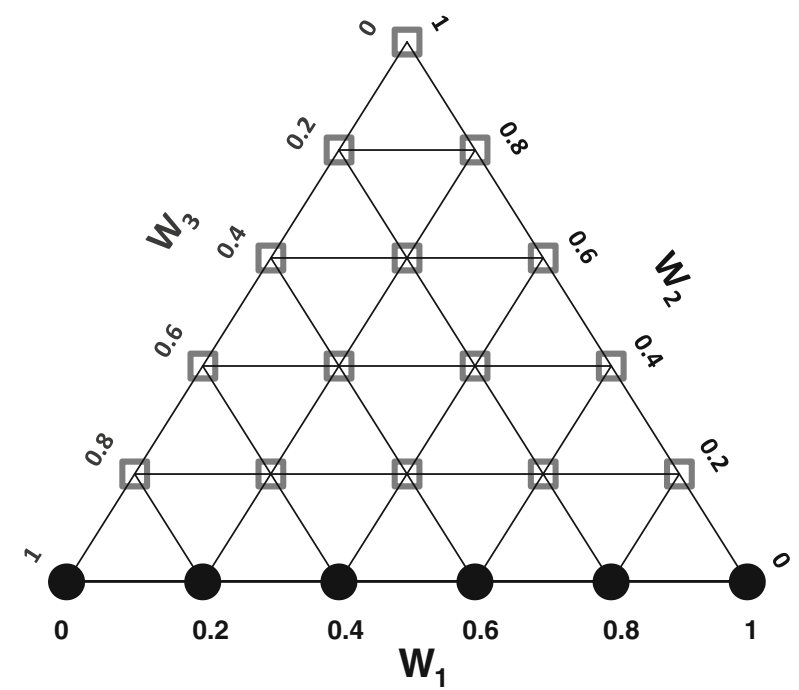

b

Swinner1

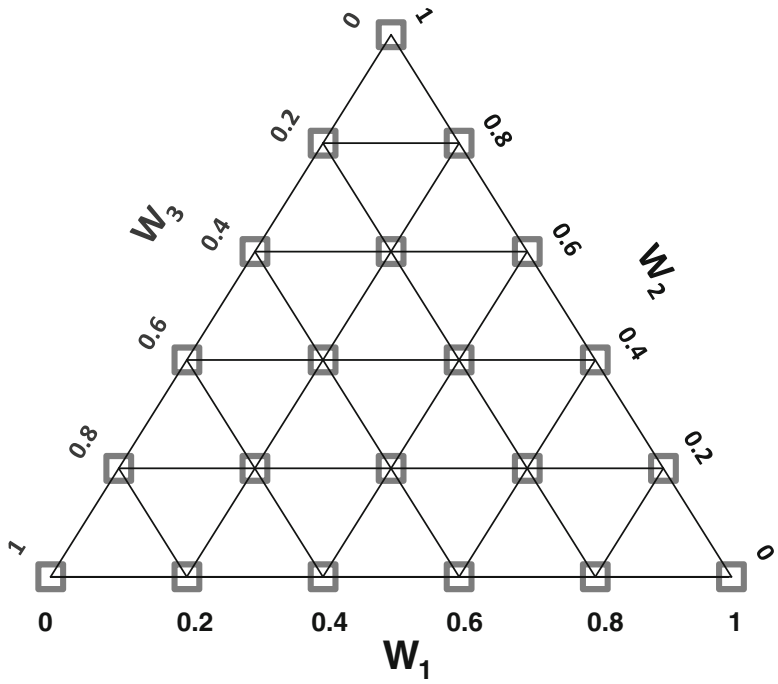

Fig. 5 Species selection depending on beliefs $(0-1)$ at $t=0\left(W^{1-3}\right)$ and decision points; periods 2 (a) and period 3 (b) under high climate change (III)

development. The optimal decision at the beginning of the first period (starting point, $t=0$ ) is based on the initial beliefs, i.e. no updating has occurred in model time. Figure 2 shows the optimal species selection for different combinations of initial beliefs in period $1(t=0)$. As follows from Table $3, S_{\text {loser }}$ is the most dominant decision alternative in this case, whereas $S_{\text {winner2 }}$ could be considered for some beliefs, but only if the decision-maker is fairly sure that climate II is the true underlying model (complete belief in medium climate change). Notice that none of the other tree species are chosen. a

OSloser OSwinner2 $\square$ Sindifferent $\square$ Swinner1

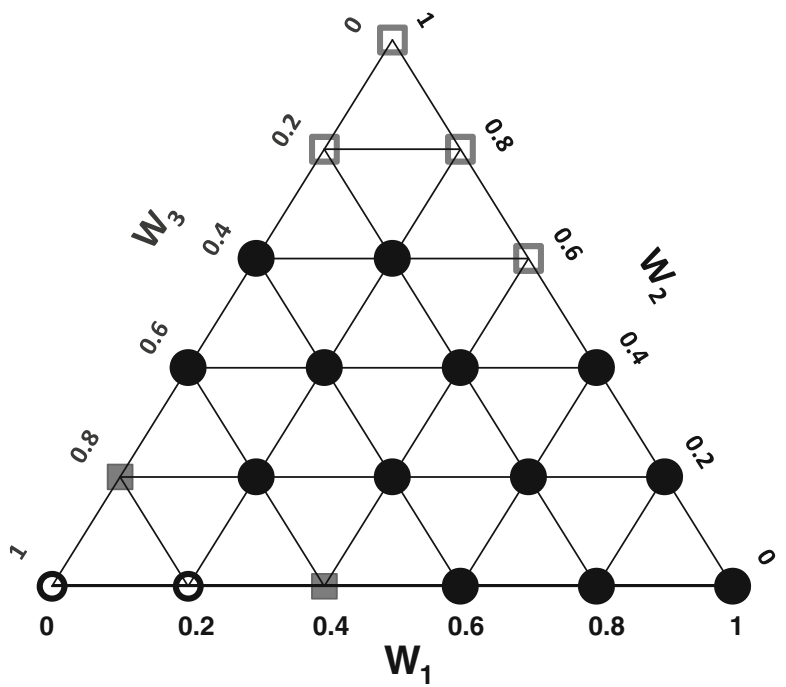

b

Swinner2 $\square$ Swinner1

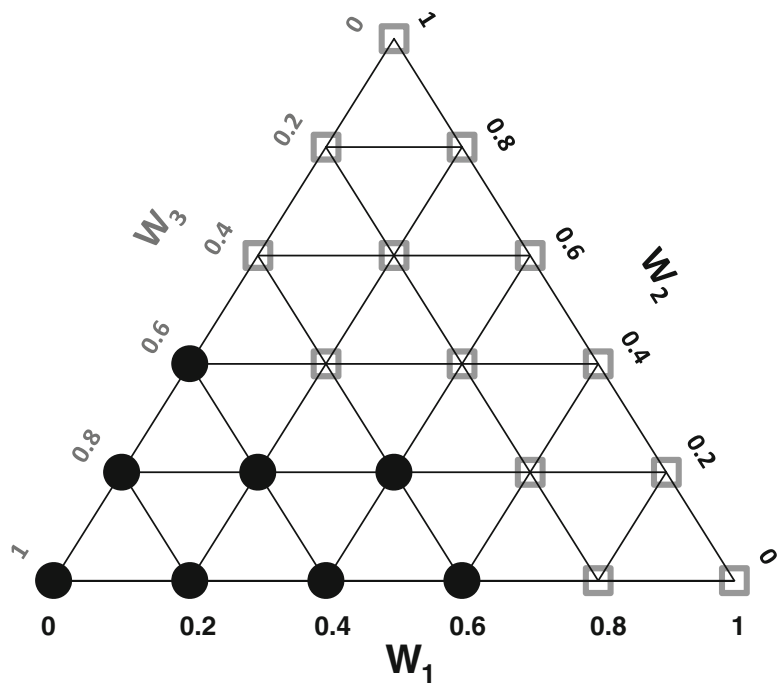

Fig. 6 Species selection for different decision points; periods 2 (a) and period 3 (b) depending only on initial beliefs (without updating over time, $W_{1-3}$ )

Figures 3, 4, and 5 show the decision in different periods after updating of initial beliefs, and depending on the initial beliefs at $t=0$, as well as depending on what climate change trajectory is true (I, II or III).

Across time, regarding climate model I where a negligible change in climate is expected, the dominating species selected would be $S_{\text {loser }}$ and $S_{\text {winner2, }}$, cf. Fig. 3. These two species respond the most positively to the moderate change scenario and provide the highest LEV from plantation projects. However, $S_{\text {loser }}$ is perceived to provide more benefits at earlier stages 
Table 4 Largest possible gain in LEV (land expectation value in 100€/ha) by making the best possible decision vs. worst decision. Best decision is made having perfect information and the worst choice is a non-adaptive decision

\begin{tabular}{|c|c|c|c|c|c|c|c|c|c|}
\hline \multirow{2}{*}{$\begin{array}{l}\text { Climate model, i } \\
\text { Year, } t\end{array}$} & \multicolumn{3}{|l|}{ I } & \multicolumn{3}{|l|}{ II } & \multicolumn{3}{|l|}{ III } \\
\hline & 2010 & 2020 & 2030 & 2010 & 2020 & 2030 & 2010 & 2020 & 2030 \\
\hline LEV best option & 4.88 & 4.81 & 4.75 & 4.81 & 5.66 & 7.5 & 4.75 & 7.5 & 9 \\
\hline Best species chosen & $S_{\text {loser* }}$ & $S_{\text {loser* }}$ & $S_{\text {winner2* }}$ & $S_{\text {loser* }}$ & $S_{\text {winner2* }}$ & $S_{\text {winner1* }}$ & $S_{\text {winner2* }}$ & $S_{\text {winner1* }}$ & $S_{\text {winner1* }}$ \\
\hline LEV worst option & 2.17 & 2.76 & 3.87 & 2.76 & 4.51 & 4.24 & 3.87 & 4.24 & 4.06 \\
\hline Worst species chosen & $S_{\text {winner1* }}$ & $S_{\text {winner1* }}$ & $S_{\text {winner1* }}$ & $S_{\text {winner1* }}$ & $S_{\text {loser* }}$ & $S_{\text {loser* }}$ & $S_{\text {winner1* }}$ & $S_{\text {loser* }}$ & $S_{\text {loser* }}$ \\
\hline Largest possible gain $(\%)$ & $121 \%$ & $74 \%$ & $23 \%$ & $74 \%$ & $25 \%$ & $77 \%$ & $23 \%$ & $77 \%$ & $122 \%$ \\
\hline
\end{tabular}

(Fig. 3a) and $S_{\text {winner2 }}$ at later stages (Fig. 3b), as climate change has progressed. $S_{\text {indifferent }}$ is an option in a very unique case and only in the second period $\left(w_{1}=0.6\right.$ and $\left.w_{2}=0.4\right)$. In the second period, both $S_{\text {loser }}$ and $S_{\text {winner2 }}$ may become the most desirable alternatives and the optimal decision depends strongly on initial beliefs. In the third period, with even moderate change well progressed, $S_{\text {winner2 }}$ dominates the decision space irrespective of the decision-maker's initial beliefs.

In case that climate model II represents the real future climate development driving simulations on which beliefs are updated, $S_{\text {winner2 }}$ becomes dominant after 10 years of climate observation, cf. Fig. 4. Once more and as shown in Fig. $4 \mathrm{a}, S_{\text {indifferent }}$ is the expected preferred decision in just two particular situations (initial beliefs $w_{1}=0.2$ and $w_{3}=0.8$, $w_{1}=0.4$ and $\left.w_{2}=0.6\right)$ and $S_{\text {winner1 }}$ comes into consideration for the first time for a high initial belief on climate II ( $w_{2}=0.6$, 0.8 , and 1$). S_{\text {loser }}$ is no longer in a dominant position in this case, where climate change will quickly reduce the beliefs in climate scenario $\mathrm{I}$, and is the preferred decision in a small area of the beliefs space $\left(w_{3}>0.6\right.$ and $\left.w_{1}=0\right)$. In the third period, after 20 years of climate observation, and the concentration of belief mass on the true, medium climate scenario (II), $S_{\text {winner1 }}$ is generally the most desirable choice, Fig. $4 \mathrm{~b}$.

In case of a high climate change (climate model III, Fig. 5), $S_{\text {winner1 }}$ occupies the decision space even from the second period (Fig. 5a) and is totally dominant in the third period Fig. 5b. $S_{\text {winner2 }}$ is still an option in the second period and in cases of some belief in climate I along with III. Neither $S_{\text {loser }}$ nor $S_{\text {indifferent }}$ could compete with $S_{\text {winner1 }}$ or $S_{\text {winner2 }}$ under highly changing climate conditions and disappear from decision space in the second and third periods.

\subsection{Optimal decision on species selection without updating}

Ignoring the value of continuous belief updating may change the optimal results and decisions on species selection. In this case, if the decisions depend only the initial, fixed beliefs and the observed performance of each species at the time of decision (thus allowing some information to be used); we reach the decision results illustrated in Fig. 6 for periods 2 (Fig. 6a) and 3 (Fig. 6b). There is an obvious difference between these decisions and the decisions based on Bayesian updating (as shown in Figs. 3, 4, and 5). As a result of the relative ranking under different scenarios, one of the most important observations is that, without updating, there will be no absolutely dominant decision (species selected) during the 30-year decision period, as in Fig. 6.

In order to get an idea of the potential gain of updating the beliefs, as compared to not updating them, we show the outcome of the worst decision, given a climate change trajectory (Table 4). If climate trajectory III is going to be realised,

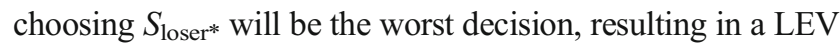
which only constitutes $45 \%$ of the outcome of the best $\left(S_{\text {winner1* }}\right)$ in the third period. Across all climate trajectories it is seen that the LEV is reduced $44-81 \%$ by choosing the worst possible species to the realised climate. Thereby, the value of the adaptive behaviour will therefore be somewhere between this and 0 , depending on how wrong the initial beliefs are.

\section{Discussion}

The contribution of our study is to highlight how the use of the Bayesian approach to analysing possible decision-making behaviours to handle climate uncertainty in forest management is a valuable addition to the study of adaptive management in general and is very likely to play a central role in the future practice and analysis of adaptive forest decision-making, in particular for irreversible or costly decisions of long-term impact. Furthermore, the Bayesian analysis has the additional benefit of presenting key findings in a format that is more easily interpreted by researchers and enables them to more easily communicate their findings to natural resource practitioners and policy makers (Ticehurst et al. 2011). For decisions with smaller long-term impacts, such as the choice of thinning intensity and the time of thinning, the value of climate-adaptive behaviour is limited and practical decision-making will therefore tend to depend more on, e.g., timber prices than on climate development (Yousefpour et al. 2012; Pukkala and Kellomäki 2012). Hauser and Possingham (2008) argue that the optimal active adaptive management may in fact be precautionary over short to medium time horizons, rather than experimental and deliberately incorporating learning. 
In our hypothetical and fairly simple case here, we have shown how a decision-maker may use new information, the observed climate stare, to revise his perceptions about the possible climate development. We show how, depending on initial beliefs, this may lead to a faster or slower convergence to a belief reflecting the actual development. In our simple model, we simplified possible climate change developments to being one out of only three illustrative alternatives. In principle, however, there is no limitation on the number of climate models that can be applied, and implementing the concept for a large number of models is a technical and computational issue only. However, it is useful to consider the extreme bounds of the climate development pathways from low change to the highest credible change in the climate state. This also applies with respect to the number of management alternatives, which can exponentially expand the solution space. To cope with this problem, dynamic programming provides a unique solution to cut the decision space (Armstrong et al. 2007; Jacobsen and Thorsen 2003; Eriksson 2006), which is providentially compatible with the Bayesian adaptive approach (McDonald-Madden et al. 2010; Probert et al. 2010). Therefore, one possible future research avenue of interest is to combine the present concept of knowledge updating based on Bayesian theory with dynamic programming to solve comprehensive forest management problems. A daunting challenge for that endeavor is, however, that the state space will need to be expanded considerable with the possible belief configurations of owners and their state dependent transition probabilities to new beliefs.

As illustrated in our results, using observed climate state, $x^{q}$, to update beliefs will make these converge towards the true climate model over time. In our hypothetical example, it takes just a decade (10 years) of observation to concentrate beliefs on the true climate model if the climate change development is very low. It takes more time (up to three decades) if a medium or high change in climate state (Fig. 1) is the true underlying driver of observations. This is due to the fact that these trajectories diverge slowly from each other, but quickly diverge from the low change scenario, and due to the considerable per period variation in climate, cf. Eq. (2). The scale of the annual variation around the climate trajectories clearly has an impact on how early and easy a decision-maker can infer which climate trajectory is most likely to be the one driving observations. In our simulations, we used a fixed rate of $\sigma_{i}=0.3$. We analysed the sensitivity of updating procedure to different rates in Appendix A and find that a higher variance $\left(\sigma_{i}=0.5\right)$ of annual deviations from the mean, $x_{i t}$, delays the time when the decision-maker become confident about what is the true underlying climate model. If the possible state space for climate development is modelled with many, close trajectories around which variance is high. On the contrary, a lower variance $\sigma_{i}=0.1$ shortens the time needed to concentrate beliefs on the true condition of climate change. An issue discussed in the climate change debate is whether climate change will increase annual variability in climate states. That would in our model correspond to a time varying $\sigma$, which could easily be implemented. Clearly, the implications would still be to reduce the tendency of decision-maker beliefs to concentrate on a specific climate trajectory.

Hauser and Possingham (2008) declare that the choice of prior weights (beliefs) for alternative hypotheses can have an important effect on future learning and consequently on the optimal decisions. Moreover, individual decision-makers would naturally interpret the results of applying different management actions through their own prior beliefs about the relative validity of different climate change models (Iverson and Perrings 2012). In our example of species selection under different climate states, $S_{\text {winner1 }}$ and $S_{\text {winner2 } 2}$ were the optimal competing decisions when a significant change in climate state was observed (see Figs. 2, 3, and 4). $S_{\text {indifferent }}$ as a climateindifferent, but moderately productive species came out as a preferred choice under very specific combinations of beliefs and true scenarios (see Figs. 3 and 4). $S_{\text {loser }}$ was modelled as sensitive to larger changes in climate, and we saw that this cause $S_{\text {loser }}$ to be a dominant optimal decision only in the earlier phases of climate change but even for moderate changes it lost its position as soon as a change in climate state was realised (see Figs. 2, 3, and 4). The productivity levels have been set artificially here, but also set to reveal generally likely differences between alternatives: Species that have been strong in past and current climates, but may suffer from significant climate change (represented by $S_{\text {loser }}$ in our model here), will be preferred by decision-makers with strong belief in no or moderate climate change, and by decision-makers that are not receptive to new information documenting climate change effects.

This way of modelling species choice of course also has limitations, and a few words may be needed to reflect on the impacts at forest level. Considering what would happen at forest level for a decision-maker adjusting his beliefs and hence decisions over several decades, we should start by acknowledging that the initial state of the forest may determine for many decades still, a dominant part of the composition. Even if relative performances of species change or are expected to change, it is rarely optimal to cut existing stands prematurely (Pukkala and Kellomäki 2012). Thus, at forest level, the composition changes only slowly in our model as the decision-maker switches preferences among possible species according to his updated beliefs (see, e.g. Yousefpour et al. 2012). Another aspect we should consider here is that we assume land quality to be homogenous, but there may be areas of a forest where the relative performance of the species will be different due to local site aspects (hydrology, wind exposure etc.) - irrespective of climate change effects. Finally, our decision-makers chose according to expected LEV, and hence ignore variation - they are essentially assumed riskneutral. It is well-known that risk aversion may lead to decisionmakers favoring some mixture, a portfolio, of species (Neuner et al., 2013). A clear challenge in this context is of course that there is little empirical knowledge on the variance and co- 
variance of species performance outcomes across climate change scenarios, and thus an optimal portfolio is not easily assessed.

For the Bayesian updating, it is not possible to estimate directly a value of waiting. However, within the frame of the analysed climate trajectories of our model, it can be shown that having the worst expectation at the third period can result in a LEV down to $45 \%$ of the one choosing the best adaptive decision. Consequently, the value of updating the system lies between 0 and $56 \%$ (Table 4 ) resulting in up to $122 \%$ more LEV than the worst i.e. non-adaptive decision. In this paper, we have not modelled the possibility of postponing the decision, i.e., the value of waiting. Schou (2013) is an example of an approach looking at the irreversibility of the decision and computed the LEV gain of waiting for novel information over time.
As a concluding remark, we highlight that the present study should be considered an inspirational basis only for many possible future developments in the field of knowledge management, climate change uncertainty and development in forest resource management. More attention is needed to investigate the benefits, the limitations and the practicability of Bayesian approaches as well as other theories for knowledge update and adaptive management of natural resources under climatic changes.

Acknowledgments and funding This study was conducted as part of the project MOTIVE 'MOdels for adapTIVE forest management' funded by the European Community's Seventh Framework Programme (FP7/ 2007-2013) under grant agreement no. 226544. JBJ and BJT further acknowledge the support of the Danish National Science foundation.

\section{Appendix A}

Table 5 Sensitivity analysis of beliefs update to observation variances

Climatic observation

\begin{tabular}{|c|c|c|c|c|c|c|c|c|c|c|c|c|c|c|c|c|c|}
\hline \multicolumn{9}{|c|}{$\sigma=0.1$} & \multicolumn{9}{|c|}{$\sigma=0.5$} \\
\hline \multicolumn{9}{|c|}{ Climate change Model Climate Change Model } & \multicolumn{9}{|c|}{ Climate change Model Climate Change Model } \\
\hline I & & & II & & & III & & & I & & & II & & & III & & \\
\hline \multicolumn{3}{|c|}{ Period } & \multicolumn{3}{|c|}{ Period } & \multicolumn{3}{|c|}{ Period } & \multicolumn{3}{|c|}{ Period } & \multicolumn{3}{|c|}{ Period } & \multicolumn{3}{|c|}{ Period } \\
\hline 1 & 2 & 3 & 1 & 2 & 3 & 1 & 2 & 3 & 1 & 2 & 3 & 1 & 2 & 3 & 1 & 2 & 3 \\
\hline 0.00 & 1.00 & 1.00 & 0.00 & 1.00 & 1.00 & 1.00 & 1.00 & 1.00 & 0.00 & 0.00 & 1.00 & 0.00 & 0.00 & 0.03 & 1.00 & 1.00 & 1.00 \\
\hline 0.00 & 0.53 & 1.00 & 0.20 & 1.00 & 1.00 & 0.80 & 1.00 & 1.00 & 0.00 & 0.00 & 0.13 & 0.20 & 0.43 & 0.99 & 0.80 & 0.92 & 1.00 \\
\hline 0.00 & 0.36 & 1.00 & 0.40 & 1.00 & 1.00 & 0.60 & 1.00 & 1.00 & 0.00 & 0.00 & 0.07 & 0.40 & 0.67 & 0.99 & 0.60 & 0.82 & 1.00 \\
\hline 0.00 & 0.27 & 1.00 & 0.60 & 1.00 & 1.00 & 0.40 & 1.00 & 1.00 & 0.00 & 0.00 & 0.05 & 0.60 & 0.82 & 1.00 & 0.40 & 0.67 & 0.99 \\
\hline 0.00 & 0.22 & 1.00 & 0.80 & 1.00 & 1.00 & 0.20 & 1.00 & 1.00 & 0.00 & 0.00 & 0.04 & 0.80 & 0.92 & 1.00 & 0.20 & 0.43 & 0.99 \\
\hline 0.00 & 0.18 & 1.00 & 1.00 & 1.00 & 1.00 & 0.00 & 1.00 & 1.00 & 0.00 & 0.00 & 0.03 & 1.00 & 1.00 & 1.00 & 0.00 & 0.00 & 0.03 \\
\hline 0.20 & 1.00 & 1.00 & 0.00 & 0.53 & 1.00 & 0.80 & 1.00 & 1.00 & 0.20 & 0.77 & 1.00 & 0.00 & 0.00 & 0.03 & 0.80 & 0.98 & 1.00 \\
\hline 0.20 & 1.00 & 1.00 & 0.20 & 1.00 & 1.00 & 0.60 & 1.00 & 1.00 & 0.20 & 0.51 & 1.00 & 0.20 & 0.37 & 0.99 & 0.60 & 0.88 & 1.00 \\
\hline 0.20 & 1.00 & 1.00 & 0.40 & 1.00 & 1.00 & 0.40 & 1.00 & 1.00 & 0.20 & 0.38 & 0.99 & 0.40 & 0.59 & 1.00 & 0.40 & 0.73 & 1.00 \\
\hline 0.20 & 1.00 & 1.00 & 0.60 & 1.00 & 1.00 & 0.20 & 1.00 & 1.00 & 0.20 & 0.30 & 0.99 & 0.60 & 0.74 & 1.00 & 0.20 & 0.48 & 0.99 \\
\hline 0.20 & 1.00 & 1.00 & 0.80 & 1.00 & 1.00 & 0.00 & 1.00 & 1.00 & 0.20 & 0.25 & 0.99 & 0.80 & 0.84 & 1.00 & 0.00 & 0.00 & 0.03 \\
\hline 0.40 & 1.00 & 1.00 & 0.00 & 0.36 & 1.00 & 0.60 & 1.00 & 1.00 & 0.40 & 0.90 & 1.00 & 0.00 & 0.00 & 0.03 & 0.60 & 0.95 & 1.00 \\
\hline 0.40 & 1.00 & 1.00 & 0.20 & 1.00 & 1.00 & 0.40 & 1.00 & 1.00 & 0.40 & 0.69 & 1.00 & 0.20 & 0.32 & 0.99 & 0.40 & 0.80 & 1.00 \\
\hline 0.40 & 1.00 & 1.00 & 0.40 & 1.00 & 1.00 & 0.20 & 1.00 & 1.00 & 0.40 & 0.56 & 1.00 & 0.40 & 0.53 & 1.00 & 0.20 & 0.55 & 0.99 \\
\hline 0.40 & 1.00 & 1.00 & 0.60 & 1.00 & 1.00 & 0.00 & 1.00 & 1.00 & 0.40 & 0.48 & 1.00 & 0.60 & 0.67 & 1.00 & 0.00 & 0.00 & 0.05 \\
\hline 0.60 & 1.00 & 1.00 & 0.00 & 0.27 & 1.00 & 0.40 & 1.00 & 1.00 & 0.60 & 0.95 & 1.00 & 0.00 & 0.00 & 0.03 & 0.40 & 0.90 & 1.00 \\
\hline 0.60 & 1.00 & 1.00 & 0.20 & 1.00 & 1.00 & 0.20 & 1.00 & 1.00 & 0.60 & 0.79 & 1.00 & 0.20 & 0.28 & 0.99 & 0.20 & 0.64 & 1.00 \\
\hline 0.60 & 1.00 & 1.00 & 0.40 & 1.00 & 1.00 & 0.00 & 1.00 & 1.00 & 0.60 & 0.67 & 1.00 & 0.40 & 0.48 & 1.00 & 0.00 & 0.00 & 0.07 \\
\hline 0.80 & 1.00 & 1.00 & 0.00 & 0.22 & 1.00 & 0.20 & 1.00 & 1.00 & 0.80 & 0.98 & 1.00 & 0.00 & 0.00 & 0.03 & 0.20 & 0.77 & 1.00 \\
\hline 0.80 & 1.00 & 1.00 & 0.20 & 1.00 & 1.00 & 0.00 & 1.00 & 1.00 & 0.80 & 0.84 & 1.00 & 0.20 & 0.25 & 0.99 & 0.00 & 0.00 & 0.13 \\
\hline 1.00 & 1.00 & 1.00 & 0.00 & 0.18 & 1.00 & 0.00 & 1.00 & 1.00 & 1.00 & 1.00 & 1.00 & 0.00 & 0.00 & 0.03 & 0.00 & 0.00 & 1.00 \\
\hline
\end{tabular}

$\sigma=$ variance $\left(\sigma^{2}\right)$ in Wiener noise process used for the realisation of climate observations

Climate Change Scenarios I-III $=$ low, medium, and high change in climate state

Periods $1-3=$ decision points at 2010, 2020, and 2030 


\section{Appendix B}

Table 6 Land expectation value (LEV) of optimal decisions based on Bayesian updating

\begin{tabular}{|c|c|c|c|c|c|c|c|c|c|c|c|}
\hline \multirow{2}{*}{\multicolumn{3}{|c|}{ Initial beliefs }} & \multirow{2}{*}{\multicolumn{3}{|c|}{$\begin{array}{l}\text { Climate I } \\
\text { Period }\end{array}$}} & \multirow{2}{*}{\multicolumn{3}{|c|}{$\begin{array}{l}\text { Climate II } \\
\text { Period }\end{array}$}} & \multirow{2}{*}{\multicolumn{3}{|c|}{$\begin{array}{l}\text { Climate III } \\
\text { Period }\end{array}$}} \\
\hline & & & & & & & & & & & \\
\hline$W_{1}$ & $W_{2}$ & $W_{3}$ & 1 & 2 & 3 & 1 & 2 & 3 & 1 & 2 & 3 \\
\hline 0 & 0 & 1 & 475 & 689 & 475 & 475 & 750 & 751 & 475 & 750 & 900 \\
\hline 0 & 0.2 & 0.8 & 470 & 567 & 475 & 470 & 590 & 750 & 470 & 748 & 900 \\
\hline 0 & 0.4 & 0.6 & 473 & 566 & 475 & 473 & 576 & 750 & 473 & 743 & 900 \\
\hline 0 & 0.6 & 0.4 & 476 & 566 & 475 & 476 & 570 & 750 & 476 & 735 & 900 \\
\hline 0 & 0.8 & 0.2 & 478 & 566 & 475 & 478 & 567 & 750 & 478 & 714 & 900 \\
\hline 0 & 1 & 0 & 481 & 566 & 475 & 481 & 566 & 750 & 481 & 566 & 900 \\
\hline 0.2 & 0 & 0.8 & 472 & 480 & 475 & 472 & 498 & 750 & 472 & 750 & 900 \\
\hline 0.2 & 0.2 & 0.6 & 474 & 470 & 475 & 474 & 535 & 750 & 474 & 747 & 900 \\
\hline 0.2 & 0.4 & 0.4 & 477 & 477 & 475 & 477 & 544 & 750 & 477 & 740 & 900 \\
\hline 0.2 & 0.6 & 0.2 & 480 & 493 & 475 & 480 & 547 & 750 & 480 & 722 & 900 \\
\hline 0.2 & 0.8 & 0 & 482 & 505 & 475 & 482 & 550 & 750 & 482 & 565 & 900 \\
\hline 0.4 & 0 & 0.6 & 476 & 480 & 475 & 476 & 471 & 750 & 476 & 750 & 900 \\
\hline 0.4 & 0.2 & 0.4 & 478 & 474 & 475 & 478 & 501 & 750 & 478 & 745 & 900 \\
\hline 0.4 & 0.4 & 0.2 & 481 & 470 & 475 & 481 & 520 & 750 & 481 & 730 & 900 \\
\hline 0.4 & 0.6 & 0 & 484 & 470 & 475 & 484 & 529 & 750 & 484 & 564 & 900 \\
\hline 0.6 & 0 & 0.4 & 480 & 480 & 475 & 480 & 475 & 750 & 480 & 750 & 900 \\
\hline 0.6 & 0.2 & 0.2 & 482 & 476 & 475 & 482 & 478 & 750 & 482 & 739 & 900 \\
\hline 0.6 & 0.4 & 0 & 485 & 473 & 475 & 485 & 502 & 750 & 485 & 562 & 900 \\
\hline 0.8 & 0 & 0.2 & 484 & 480 & 475 & 484 & 478 & 750 & 484 & 749 & 900 \\
\hline 0.8 & 0.2 & 0 & 487 & 477 & 475 & 487 & 470 & 750 & 487 & 557 & 900 \\
\hline 1 & 0 & 0 & 488 & 480 & 475 & 488 & 480 & 750 & 488 & 473 & 900 \\
\hline Ave & rage & & 479 & 510 & 475 & 479 & 533 & 750 & 479 & 686 & 900 \\
\hline Std. & dev. & & 5 & 56 & 0 & 5 & 63 & 0 & 5 & 92 & 0 \\
\hline
\end{tabular}

Initial beliefs beliefs on climate models (I-III) at first decision point i.e. 2010; Climates I-III Climate models for low, medium and high change in climate state; Periods 1-3 decision points at 2010-2030 with 10 years interval; $W_{1}-W_{3}$ belief on climate models I-III $[0,1]$; Std. dev. Standard deviation (of average outcome based on different set of initial beliefs)

\section{References}

Allen M, Stott P, Mitchell J, Schnur R, Delworth T (2000) Quantifying the uncertainty in forecasts of anthropogenic climate change. Nature 407:617-620

Armstrong D, Castro I, Griffiths R (2007) Using adaptive management to determine requirements of re-introduced populations: the case of the New Zealand hihi. J Appl Ecol 44:953-962

Bayes T, Price M (1763) An essay towards solving a problem in the doctrine of chances. Philos Trans R Soc London 53:370-418

Bolte A, Ammer C, Loef M, Madsen P, Nabuurs G, Schall P, Spathelf P, Rock J (2009) Adaptive forest management in central Europe: climate change impacts, strategies and integrative concept. Scand J Forest Res 24:473-482

Crome F, Thomas M, Moore LA (1996) A novel Bayesian approach to assessing impacts of rain forests logging. Ecol Appl 6:1104-1123

Eriksson LO (2006) Planning under uncertainty at the forest level: a systems approach. Scand J Forest Res 21:111-117

Hanewinkel M, Hummel S, Albrecht A (2011) Assessing natural hazards in forestry for risk management: a review. Eur J Forest Res 130:329-351

Hauser C, Possingham H (2008) Experimental or precautionary? Adaptive management over a range of time horizons. J Appl Ecol 45:72-81

Hildebrandt P, Knoeke T (2011) Investment decisions under uncertainty - a methodological review on forest science studies. Forest Policy Econ 13:1-15

Iverson T, Perrings C (2012) Precaution and proportionality in the management of global environmental change. Global Environ Chang 22:161-177

Jacobsen JB, Thorsen BJ (2003) A Danish example of optimal thinning strategies in mixed-species forest under changing growth conditions caused by climate change. Forest Ecol Manag 180:375-388

Kangas J, Store R, Leskinen P, Mehtaetalo L (2000) Improving the quality of landscape ecological forest planning by utilizing advanced decision-support tools. Forest Ecol Manag 132:157-171

McDonald-Madden E, Probert W, Hauser C, Runge M, Possingham H, Jones ME, Moore JL, Rout TM, Vesk PA, Wintle BA (2010) Active adaptive conservation of threatened species in the face of uncertainty. Ecol Appl 20:1476-1489

Neuner S, Beinhofer B, Knoke T (2013) The optimal tree species composition for a private forest enterprise - applying the theory of portfolio selection. Scand J For Res 28:28-48

Prato T (2000) Multiple attributes Bayesian analysis of adaptive ecosystem management. Ecol Model 133:181-193

Probert W, Hauser C, McDonald-Madden E, Michael C, Baxter P, Possingham $\mathrm{H}$ (2010) Managing and learning with multiple models: objectives and optimization algorithms. Biol Conserv 144:1237-1245

Pukkala T, Kellomäki S (2012) Anticipatory vs adaptive optimization of stand management when tree growth and timber prices are stochastic. Forestry $85: 463-472$

Scherrer S, Appenzeller C, Liniger M, Schär C (2005) European temperature distribution changes in observations and climate change scenarios. Geophys Res Lett 32, L19750. doi:10.1029/2005GL024108

Schou E (2013) Transformation to near-natural forest management, Climate change and uncertainty. Dissertation, University of Copenhagen

Spiecker H (2003) Silvicultural management in maintaining biodiversity and resistance of forests in Europe-temperate zone. J Environ Manage 67:55-65

Sykes M, Prentice C (1996) Climate change, tree species distributions and forest dynamics. A case study in the mixed conifer/Northern hardwoods zone of Northern Europe. Clim Change 34:161-177

Ticehurst J, Curtis A, Merritt W (2011) Using Bayesian networks to complement conventional analyses to explore landholder management of native vegetation. Environ Model Softw 26:52-65

Tomar AS, Ranade DH (2002) Predicting cumulative rainfall deficits by Gompertz Growth Model. Ind J Soil Conserv 30:101-103

Yousefpour R, Hanewinkel M (2009) Modelling of forest conversion planning with an adaptive simulation-optimization approach and simultaneous consideration of the values of timber, carbon and biodiversity. Ecol Econ 68:1711-1722

Yousefpour R, Jacobsen JB, Thorsen BJ, Meilby H, Hanewinkel M, Oehler K (2012) A review of decision-making approaches to handle uncertainty and risk in adaptive forest management under climate change. Ann For Sci 69:1-15 